\title{
Hyperbilirubinemia as a Predictor of Appendiceal Perforation: A Systematic Review and Diagnostic Test Meta-Analysis
}

\author{
Paschalis Gavriilidis ${ }^{\mathrm{a}, \mathrm{d}}$, Nicola de'Angelis ${ }^{\mathrm{b}}$, John Evans ${ }^{\mathrm{a}}$, \\ Salomone Di Saverio ${ }^{c}$, Peter Kang ${ }^{\mathrm{a}}$
}

\begin{abstract}
Background: Misdiagnosis of the severity of acute appendicitis may lead to perforation and can consequently result in increased morbidity and mortality. In this study, the role of hyperbilirubinemia as a predictor of perforation is assessed by performing a meta-analysis of diagnostic accuracy.
\end{abstract}

Methods: A systematic search of the literature published over the past 20 years was performed using the EMBASE, PubMed, Cochrane library, and Google Scholar databases.

Results: Low values of sensitivity, specificity, and diagnostic odds ratio (DOR) were detected: 0.21 (95\% confidence interval (CI): 0.13 0.30 , standard error $(\mathrm{SE})=0.43), 0.27(95 \% \mathrm{CI}: 0.15-0.43, \mathrm{SE}=0.73)$, and $0.10(95 \% \mathrm{CI}: 0.3-0.28, \mathrm{SE}=0.05)$, respectively. The positive likelihood ratio (PLR) was low (0.29 (95\% CI: $0.27-0.91, \mathrm{SE}=0.76)$ ), whereas the negative likelihood ratio (NLR) was high $(2.88$ (95\% CI: $1.66-5.14, \mathrm{SE}=0.10)$ ). The hierarchical summary receiver operating characteristic curve was positioned towards the lower right corner, and the area under the curve was 0.19 , both indicating a low level of overall accuracy and discrimination. Compared with the PLR, the negative inverse likelihood ratio $\left(1 / \mathrm{LR}^{-}\right)$indicated that a positive result has a greater impact on the odds of disease than does a negative result.

Conclusions: Hyperbilirubinemia alone is not a reliable tool to predict perforation. Future studies should investigate whether the combined predictive values of bilirubin, C-reactive protein (CRP), and white blood cells are a more effective diagnostic tool.

Keywords: Perforated appendicitis; Complicated appendicitis; Hy-

Manuscript submitted December 15, 2018, accepted January 14, 2019

aDepartment of General and Colorectal Surgery, Northampton General Hospital NHS Trust, Northampton, UK

${ }^{b}$ Department of Digestive Surgery, Henri Mondor University Hospital, Creteil, France

'Department of Colorectal Surgery, Cambridge University Hospitals NHS Foundation Trust, Addenbrooke's Hospital, Hills Road, Cambridge, UK

${ }^{\mathrm{d} C}$ Corresponding Author: Paschalis Gavriilidis, Department of General and Colorectal Surgery, Northampton General Hospital NHS Trust, Northampton NN1 5BD, UK. Email: pgavrielidis@yahoo.com

doi: https://doi.org/10.14740/jocmr3724 perbilirubinemia

\section{Introduction}

Acute appendicitis may present with various symptoms, signs, and laboratory results, and therefore, diagnosis is not easy [1]. The following scores are useful in the differential diagnosis of acute appendicitis in patients presenting with right iliac fossa pain: the Alvarado score, modified Alvarado score, Raja 1steri Pengiran Anak Saleha Appendicitis score designed specifically for Asian patients, and the Appendicitis Inflammatory Response (AIR) score based on symptoms (nausea, vomiting, duration of pain, and migratory pain), signs (fever, localized tenderness, and rebound tenderness), and laboratory results (leukocytosis, neutrophilia, and increased C-reactive protein (CRP)). Yet, these scoring systems cannot distinguish between uncomplicated and complicated appendicitis [2-4].

It is reported that the mortality rate of uncomplicated appendicitis is $0.3 \%$; however, this increases to $6 \%$ in perforated cases [5]. In the diagnosis of acute appendicitis, the supplemental roles of ultrasound and computed tomography scans are essential. However, they have a low sensitivity in detecting perforated appendicitis $[6,7]$. Therefore, the predictive ability of hyperbilirubinemia in the diagnosis of perforated appendicitis, as a supplementary tool to clinical examination and imaging studies, is worthy of investigation.

To date, many authors of retrospective studies have evaluated hyperbilirubinemia as a prognostic factor for appendiceal perforation. It is reported that hyperbilirubinemia commonly occurs in patients with septic conditions and in cases of complicated appendicitis $[8,9]$.

These retrospective studies, which are based on small population groups, suggest that hyperbilirubinemia may be a useful predictor of perforated appendicitis.

Our study aims to investigate hyperbilirubinemia as a predictive factor of appendiceal perforation, using a meta-analysis of diagnostic test accuracy.

\section{Methods}

The preferred reporting items for systematic reviews and me- 
ta-analyses (PRISMA) statement checklist was followed in this study [10].

\section{Literature search}

Using the search terms in both free text and MESH terms (perforated appendicitis, appendiceal perforation, hyperbilirubinemia, jaundice, bilirubin, elevated bilirubin, complicated appendicitis, and appendix), a systematic search of the literature published over the past 20 years was performed, through the EMBASE, PubMed, Cochrane library, and Google Scholar databases. A grey literature search at the clinicaltrials.gov website was also undertaken. The references in the retrieved articles were manually checked for further studies. Disagreements between the authors were resolved through consensus-based discussions.

\section{Study selection, inclusion, and exclusion criteria}

Our study included solely studies that compared the diagnostic test accuracy of elevated bilirubin in assessing perforated appendicitis. Included studies were required to meet the following criteria: 1) Reporting all-comers population with appendicitis and bilirubin measurements; 2) Comparing a cohort of perforated cases with non-complicated cases; and 3) In the event of multiple publications by the same institution, only the most recent publication was included.

Case reports and studies in which it was impossible to clearly calculate the outcomes were excluded from the analysis.

\section{Data extraction and outcomes}

Two reviewers (PG and PK) independently extracted the following summary data for the included studies: true positives, false positives, true negatives, and false negatives.

\section{Definitions}

Hyperbilirubinemia was considered to be any value of total bilirubin serum above $1 \mathrm{mg} / \mathrm{dL}$ or $20.5 \mu \mathrm{mol} / \mathrm{L}$. Only patients with histological findings of perforated appendicitis were included in the study.

The sensitivity of a test was defined as the proportion of patients with disease and positive histological findings of perforated appendicitis and hyperbilirubinemia. The positive likelihood ratio (PLR) is the estimation of the probability of how much more likely a positive test is to be found in a patient with perforation, as opposed to without perforation. The negative likelihood ratio (NLR) is the probability of how much more likely a negative test is to be found in a patient with perforation, as opposed to without perforation. The diagnostic odds ratio (DOR) was defined as the ratio of the odds of positive findings in a participant with disease relative to positive find- ings in a participant without disease.

\section{Statistical analyses}

All statistical analyses were performed using both STATA software (version 15, StataCorp LP, College Station, TX, USA) and R software (http://meta-analysis-with-r.org). The methodological quality of all studies was assessed with the Quality Assessment of Diagnostic Accuracy Studies (QUADAS) tool [11, 12].

The following measures of test accuracy were estimated for each study: sensitivity, specificity, PLR, NLR, and DOR.

Data analysis was based on the hierarchical summary receiver operating characteristic (HSROC) and the bivariate model, which were used to account for the correlation between sensitivity and specificity. Heterogeneity between studies was estimated by comparing the $95 \%$ confidence region and $95 \%$ prediction region. The influence of each study on the model parameters was estimated with Cook's distance [13].

The inverse of the NLR $\left(1 / \mathrm{LR}^{-}\right)$is also estimated, because larger values indicate a more accurate test. Additionally, comparison of this with the PLR can indicate whether a positive or negative test result has a greater impact on the odds of disease [13].

Positive and negative LRs were used to characterize the clinical utility of the test. It is reported that LR $=1$ means that the post-test probability is equal to the pretest probability, and thus a clinically useful test can be one with high PLR $(>5$, good in ruling in disease) and with low NLR $(<0.2$, good in ruling out disease) [14].

\section{Results}

\section{Search strategy and characteristics of included studies}

Twenty studies were included from an initial pool of 196, comprising a total of 8,751 cases, of which 6,235 (71\%) had histologically confirmed acute appendicectomy and 1,343 (15\%) had perforated appendicitis; 12 were retrospective studies and eight prospective non-randomized studies (Table 1, Fig. 1) [5, 15-17-33]. Three articles were excluded because they compared more than one test. The negative appendicectomy rate was $29 \%$. Language or region restrictions were not applied to the systematic search.

\section{Diagnostic accuracy measures}

Pooled estimates for sensitivity and specificity were $0.21(95 \%$ confidence interval $(\mathrm{CI}): 0.13-0.30$, standard error $(\mathrm{SE})=$ 0.43 ), and 0.27 (95\% CI: $0.15-0.43, \mathrm{SE}=0.73)$, respectively (Fig. 2). Pooled estimates for PLR and NLR were $0.29(95 \%$ CI: $0.27-0.91, \mathrm{SE}=0.76)$, and $2.88(95 \% \mathrm{CI}: 1.66-5.14, \mathrm{SE}$ $=0.10)$, respectively (Table1). The DOR was 0.10 (95\% CI: $0.3-0.28, \mathrm{SE}=0.05)$. The inverse of the negative likelihood ratio $\left(1 / \mathrm{LR}^{-}\right)$was $0.34(0.19-0.61)(\mathrm{SE}=0.10)$.

The HSROC curve was positioned towards the lower right 


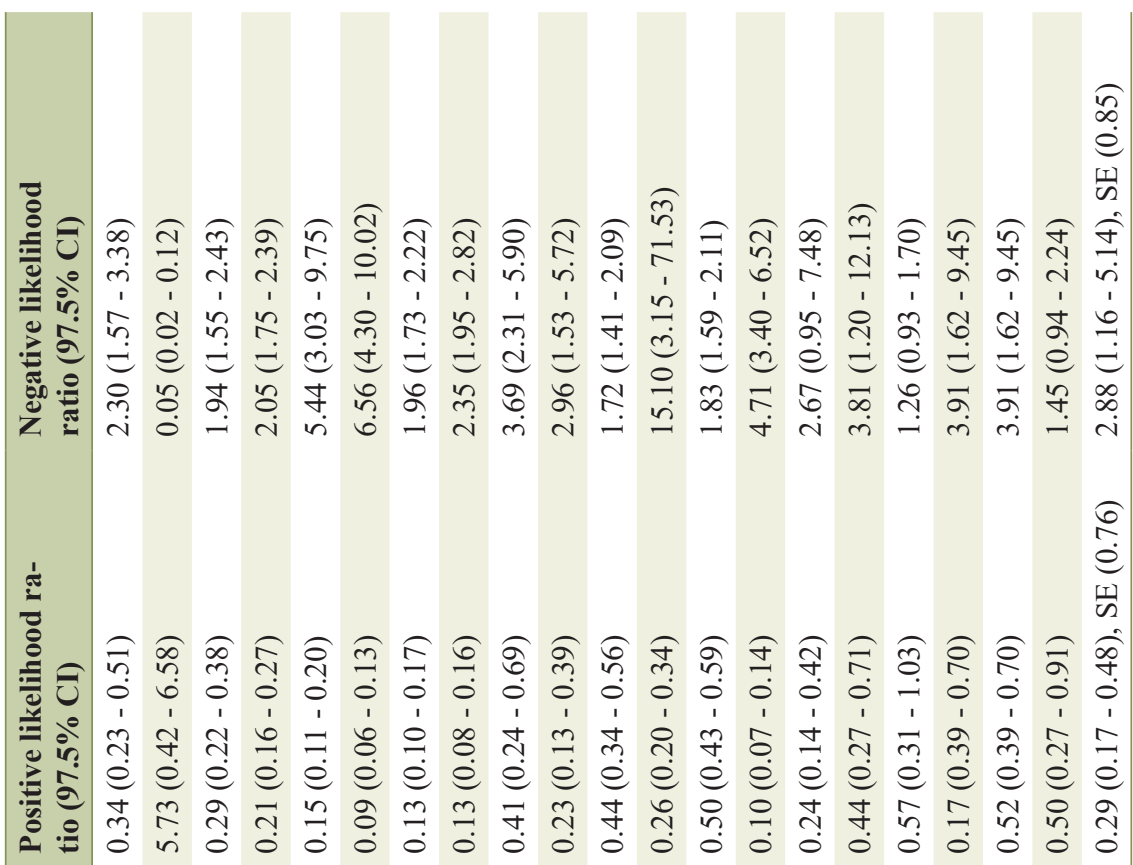

焉

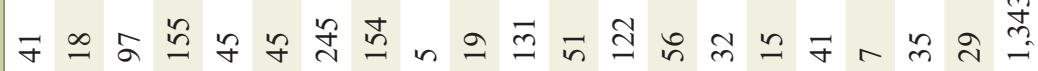

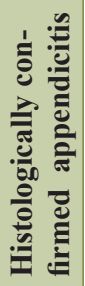

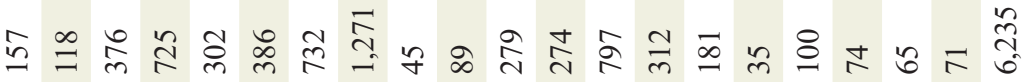




\section{PRISMA 2009 Flow Diagram}
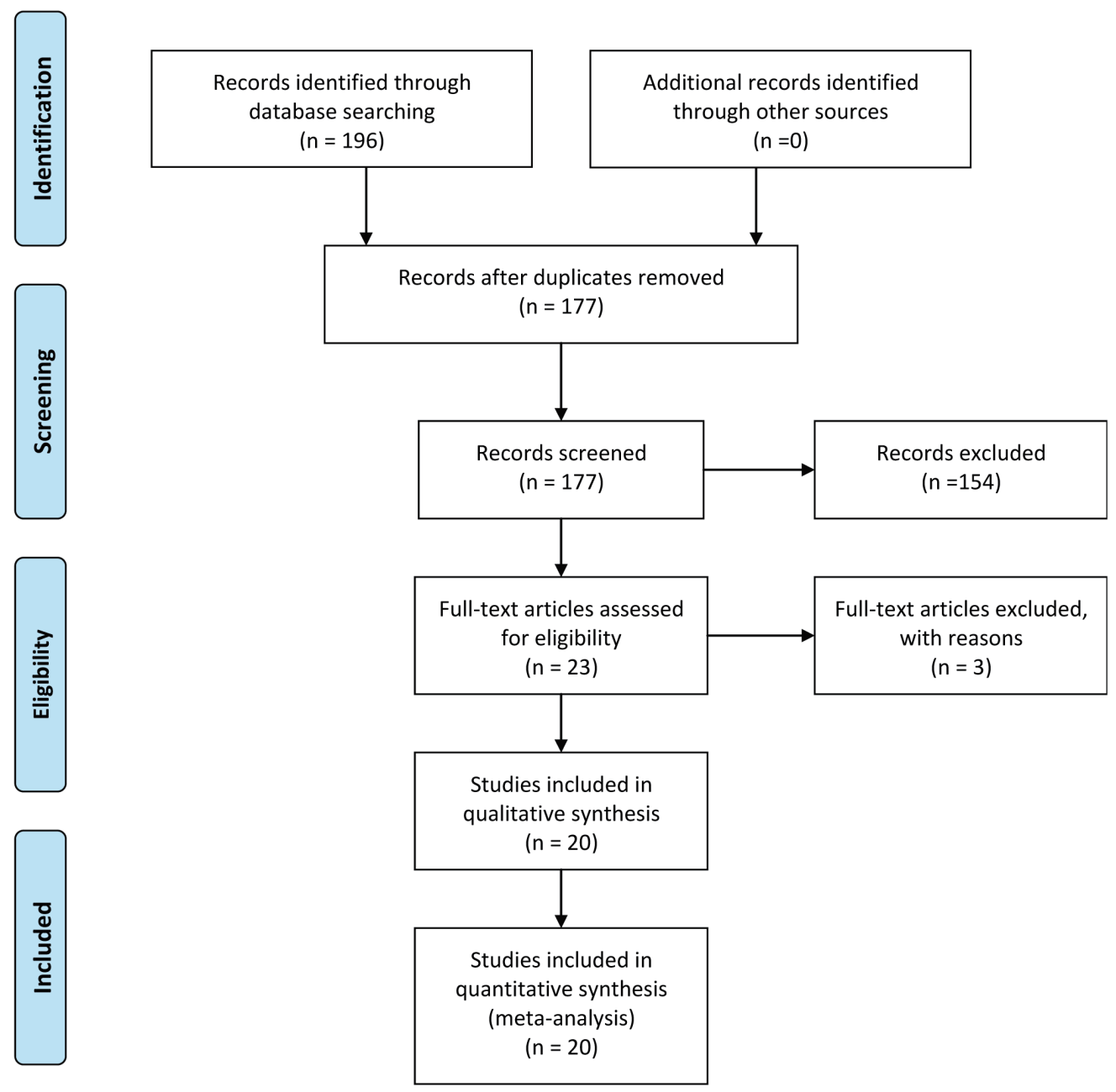

Figure 1. Flow diagram of the search strategy.

corner; the area under the curve (AUC) was 0.19 (Fig. 3).

\section{Study quality and sensitivity analysis}

Generally, the quality of the included studies was poor; none fulfilled all items on the QUADAS checklist, and none reported the diagnostic accuracy as required by the STARD guidelines $[11,34]$. Moreover, none of the outcome assessors were blinded.

Cook's distance statistic, which is a measure of the influence of a study on the model parameters detected, had only one outlier which was the study of Khan [16]. However, this study did not influence the summary estimates (Fig. 4). Investigation of the effect of individual studies on the model, by refitting the model and leaving out each study, did not demonstrate any impact on the summary estimates.

The prediction region compared to confidence region demonstrated a visual representation of high between-study heterogeneity (Fig. 3).

\section{Discussion}

Our study aims to determine whether hyperbilirubinemia is a reliable prognostic factor of appendiceal perforation. Pooled estimates of low values of sensitivity demonstrate that hyperbilirubinemia is not a useful rule-out test. In addition, the insufficiently high value of the PLR characterizes hyperbilirubinemia as a non-reliable rule-in test of perforated appendicitis. The PLR and NLR were 0.29 and 2.88, respectively. Considering that the values of PLR $>5$ and NLR $<0.2$ char- 


\begin{tabular}{|c|c|c|c|c|c|c|c|c|}
\hline Study & TP & FP & $\mathbf{F N}$ & TN & Sensitivity $(95 \% \mathrm{Cl})$ & Specificity $(95 \% \mathrm{Cl})$ & Sensitivity $(95 \% \mathrm{Cl})$ & Specificity $(95 \% \mathrm{Cl})$ \\
\hline Estrada 2007 & 23 & 36 & 18 & 80 & $0.56[0.40,0.72]$ & $0.69[0.60,0.77]$ & $\longrightarrow-$ & $\rightarrow$ \\
\hline Khan 52008 & 87 & 0 & 19 & 4 & $0.82[0.73,0.89]$ & $1.00[0.40,1.00]$ & $\rightarrow-$ & \\
\hline Sand 2009 & 73 & 60 & 45 & 365 & $0.62[0.52,0.71]$ & $0.86[0.82,0.89]$ & $\rightarrow$ & $\Rightarrow$ \\
\hline Kaser 2010 & 59 & 127 & 96 & 443 & $0.38[0.30,0.46]$ & $0.78[0.74,0.81]$ & $\rightarrow$ & $=$ \\
\hline Atahan 2011 & 36 & 49 & 9 & 257 & $0.80[0.65,0.90]$ & $0.84[0.79,0.88]$ & - & $=$ \\
\hline Emmanuel 2011 & 27 & 113 & 18 & 314 & $0.60[0.44,0.74]$ & $0.74[0.69,0.78]$ & $\rightarrow-$ & $=$ \\
\hline Hong 2012 & 62 & 149 & 135 & 849 & $0.31[0.25,0.38]$ & $0.85[0.83,0.87]$ & - & - \\
\hline Chaudhary 2013 & 8 & 30 & 0 & 12 & $1.00[0.63,1.00]$ & $0.29[0.16,0.45]$ & -5 & $\rightarrow-$ \\
\hline D'Souza 2013 & 14 & 16 & 6 & 74 & $0.70[0.46,0.88]$ & $0.82[0.73,0.89]$ & $\longrightarrow-$ & $\rightarrow$ \\
\hline McGowan 2013 & 85 & 107 & 69 & 1010 & $0.55[0.47,0.63]$ & $0.90[0.89,0.92]$ & $\rightarrow$ & - \\
\hline Nomura 2013 & 67 & 82 & 64 & 197 & $0.51[0.42,0.60]$ & $0.71[0.65,0.76]$ & $\rightarrow-$ & 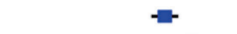 \\
\hline Socea 2013 & 45 & 28 & 1 & 136 & $0.98[0.88,1.00]$ & $0.83[0.76,0.88]$ & & $\rightarrow$ \\
\hline Chambers 2015 & 151 & 246 & 146 & 327 & $0.51[0.45,0.57]$ & $0.57[0.53,0.61]$ & $\rightarrow$ & \\
\hline Muller 2015 & 27 & 121 & 29 & 315 & $0.48[0.35,0.62]$ & $0.72[0.68,0.76]$ & - & \\
\hline Saxena 2015 & 30 & 5 & 2 & 154 & $0.94[0.79,0.99]$ & $0.97[0.93,0.99]$ & & \\
\hline Eren 2016 & 24 & 10 & 17 & 90 & $0.59[0.42,0.74]$ & $0.90[0.82,0.95]$ & $\longrightarrow$ & $\rightarrow$ \\
\hline Shahabuddin 2016 & 13 & 12 & 2 & 23 & $0.87[0.50,0.98]$ & $0.66[0.48,0.81]$ & & $\longrightarrow-$ \\
\hline Abouzeid 2017 & 7 & 51 & 0 & 36 & $1.00[0.59,1.00]$ & $0.41[0.31,0.52]$ & & $\rightarrow-$ \\
\hline Cheekuri 2017 & 31 & 27 & 4 & 38 & $0.89[0.73,0.97]$ & $0.58[0.46,0.71]$ & $\rightarrow-$ & $\rightarrow-$ \\
\hline Vineed 2017 & 19 & 9 & 10 & 62 & $0.66[0.46,0.82]$ & $0.87[0.77,0.94]$ & $\Rightarrow$ & - 눌 \\
\hline & & \multirow{2}{*}{\multicolumn{3}{|c|}{ Coef }} & \multirow{2}{*}{\multicolumn{2}{|c|}{ SE }} & \multirow{2}{*}{$95 \% \mathrm{Cl}$} & \\
\hline \multirow{2}{*}{\multicolumn{2}{|c|}{ Summary pt. }} & & & & & & & \\
\hline & & \multirow{2}{*}{\multicolumn{3}{|c|}{.2107219}} & & & \\
\hline $\mathrm{Se}$ & & & & & .0435 & 183 & .1378316 & .308372 \\
\hline Sp & & \multicolumn{3}{|c|}{.2735993} & \multicolumn{2}{|c|}{0736077.} & .1541596 & .4376926 \\
\hline DOR & & \multicolumn{3}{|c|}{.1005584} & \multicolumn{2}{|c|}{.0535} & .0354449 & .2852874 \\
\hline $\mathrm{LR}^{+}$ & & \multicolumn{3}{|c|}{.2900904} & \multicolumn{2}{|c|}{.0765516} & .1729462 & .4865817 \\
\hline $\mathrm{LR}^{-}$ & & \multicolumn{3}{|c|}{2.884795} & \multicolumn{2}{|c|}{.8522124} & 1.616803 & 5.147222 \\
\hline $1 / \mathrm{LR}^{-}$ & & \multicolumn{3}{|c|}{.346645} & \multicolumn{2}{|c|}{.1024042} & 1942796 & .6185045 \\
\hline
\end{tabular}

Figure 2. Forest plot demonstrating sensitivity and specificity. coef: coefficient; SE: standard error; Cl: confidence interval; Se: sensitivity; Sp: specificity; DOR: diagnostic odds ratio; LR+: positive likelihood ratio; LR*: negative likelihood ratio; 1/LR-: inverse negative likelihood ratio.

acterize the tests of clinical usefulness, we can conclude that hyperbilirubinemia could not be a test of high clinical usefulness. However, the negative inverse likelihood ratio (1/LR-) indicated that a positive result has a greater impact on the odds of disease than does a negative result, when compared with the PLR. This finding may justify the usefulness of hyperbilirubinemia when it is detected in clinically confirmed appendicitis, alongside other positive laboratory results. In such cases, the surgeon should consider that there is an increased probability for perforation and thus, it would be wise to proceed with an emergency intervention rather than with a planned one.

The HSROC, which is used to account for the correlation between sensitivity and specificity, was positioned towards the lower right corner. In addition, the AUC was of low value. Thus, both findings demonstrate that the test is of low overall accuracy and discriminatory ability.

Using the Cook's distance measure did not detect particularly influential studies; the only outlier was the study by Khan [16], which did not influence the pooled estimates (Fig. 4). Furthermore, investigation of the effect of individual studies on the model by refitting the model and leaving out each study did not demonstrate any impact on the summary estimates.

To our best knowledge, this is the first meta-analysis that includes 20 studies. A previous paper with only eight studies concluded that although hyperbilirubinemia alone is not a strong predictor of perforation, it can be included in the diagnostic process as a supplementary tool [35]. The results of the current study demonstrated a poorer performance of hyperbilirubinemia. There are several possible reasons for the discrepancies in the results of the present and previous studies. First, the previous study's total sample was half that of the present study. Second, studies that were published after the publication of the previous meta-analysis demonstrated worse results than those of the studies included in the previous study. Therefore, an underpowered sample, national and institutional characteristics, and selection bias may have influenced the results. The results of the present study should be interpreted within its limitations, as the overall quality of the included studies was poor and the studies were conducted at single centers. Therefore, national and institutional characteristics, performance, attrition, and selection bias may have influenced the results. Furthermore, another source of bias could be the high between-study heterogeneity (Fig. 3).

\section{Conclusions}

Hyperbilirubinemia alone has a low overall accuracy to diagnose an anticipated perforation. However, in cases with clinically confirmed appendicitis when elevated bilirubin appears besides other positive laboratory results, complicated appendi- 


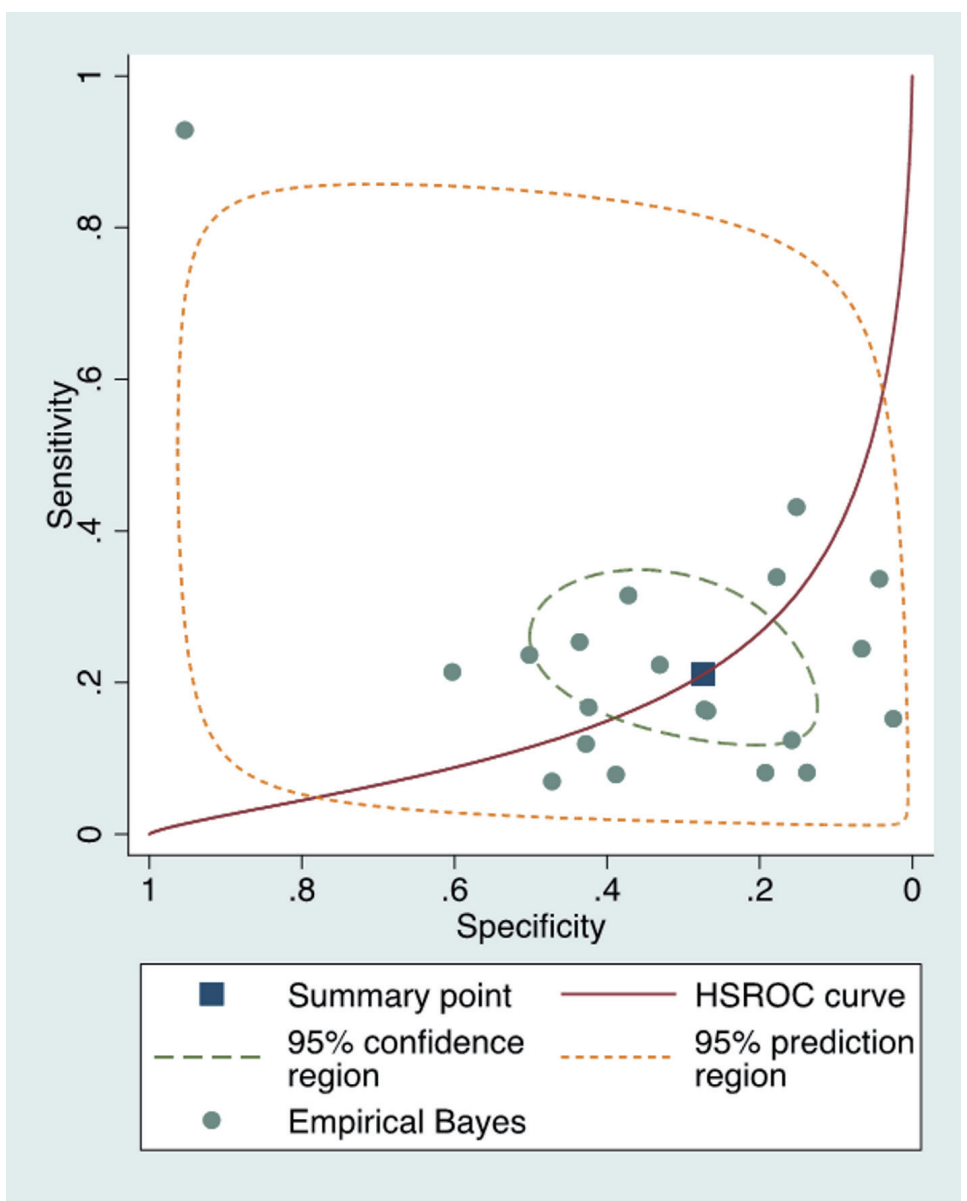

Figure 3. Plot showing the hierarchical summary receiver operating characteristic (HSROC) curve and summary operating point positioned towards the lower right angle. Obvious visual discrepancy of the covered areas of the confidence and prediction intervals indicating high between-studies heterogeneity.
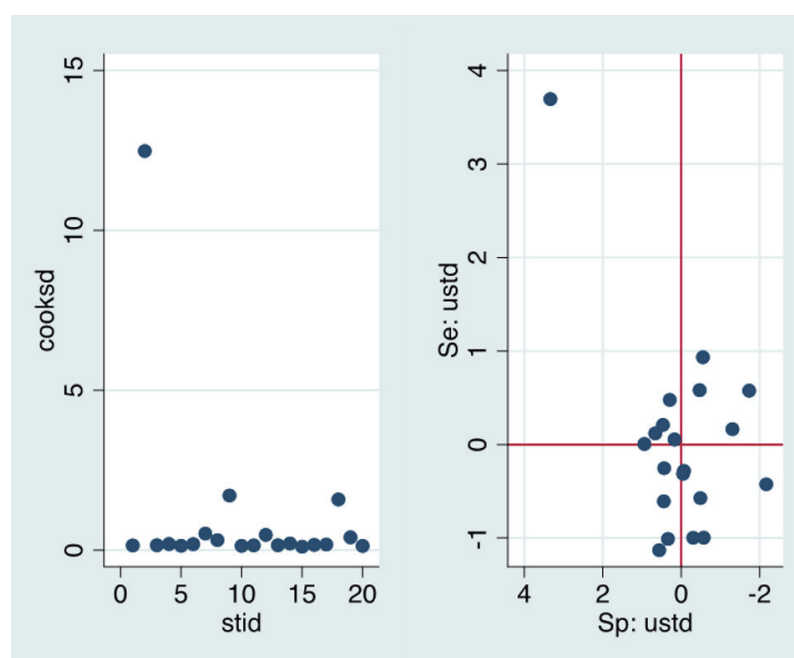

Figure 4. Left panel: Cook's distance showing one outlier (Khan's study). Right panel: standardized residuals showing one outlier (Khan's study). cooksd: Cook's standard deviation; stid: study identity; Se:ustd = sensitivity standard deviation; Sp:ustd = specificity standard deviation. citis is more likely to be diagnosed. Based on these data and in the presence of hyperbilirubinemia, the treating surgeons may prefer to proceed with immediate emergency surgery rather than pursuing non-operative management and expectant treatment further and delaying the decision of going to theatre for an appendicectomy, given the likelihood of complicated appendicitis and the risk of developing perforated appendicitis. Therefore, future studies that are adequately powered and report their results of diagnostic accuracy based on STARD guidelines are urgently needed. They should investigate whether the combined predictive value of bilirubin, CRP, and white blood cells would be a more effective diagnostic tool for surgeons.

\section{Conflict of Interest}

The authors declare no conflict of interest

\section{Ethical Approval}

This study does not contain any studies with human partici- 
pants or animals performed by any of the authors.

\section{References}

1. Petroianu A. Diagnosis of acute appendicitis. Int J Surg. 2012;10(3):115-119.

2. Alvarado A. A practical score for the early diagnosis of acute appendicitis. Ann Emerg Med. 1986;15(5):557-564.

3. Kalan M, Talbot D, Cunliffe WJ, Rich AJ. Evaluation of the modified Alvarado score in the diagnosis of acute appendicitis: a prospective study. Ann R Coll Surg Engl. 1994;76(6):418-419.

4. Chong CF, Thien A, Mackie AJ, Tin AS, Tripathi S, Ahmad MA, Tan LT, et al. Comparison of RIPASA and Alvarado scores for the diagnosis of acute appendicitis. Singapore Med J. 2011;52(5):340-345.

5. Sand M, Bechara FG, Holland-Letz T, Sand D, Mehnert G, Mann B. Diagnostic value of hyperbilirubinemia as a predictive factor for appendiceal perforation in acute appendicitis. Am J Surg. 2009;198(2):193-198.

6. Rybkin AV, Thoeni RF. Current concepts in imaging of appendicitis. Radiol Clin North Am. 2007;45(3):411-422, vii.

7. Bixby SD, Lucey BC, Soto JA, Theysohn JM, Ozonoff A, Varghese JC. Perforated versus nonperforated acute appendicitis: accuracy of multidetector CT detection. Radiology. 2006;241(3):780-786.

8. Johnson AM. Sepsis and jaundice. Pediatrics. 1993;91(5):1018.

9. Whitehead MW, Hainsworth I, Kingham JG. The causes of obvious jaundice in South West Wales: perceptions versus reality. Gut. 2001;48(3):409-413.

10. Moher D, Liberati A, Tetzlaff J, Altman DG, The PRISMA Group. Preferred reporting items for systematic reviews and meta-analyses: the PRISMA statement. PLoS Med. 2009;6(7):e1000097.

11. Whiting PF, Weswood ME, Rutjes AW, Reitsma JB, Bossuyt PN, Kleijnen J. Evaluation of QUADAS, a tool for the quality assessment of diagnostic accuracy studies. BMC Med Res Methodol. 2006;6:9.

12. Leeflang MM, Deeks JJ, Gatsonis C, Bossuyt PM, Cochrane Diagnostic Test Accuracy Working G. Systematic reviews of diagnostic test accuracy. Ann Intern Med. 2008;149(12):889-897.

13. Moses LE, Shapiro D, Littenberg B. Combining independent studies of a diagnostic test into a summary ROC curve: data-analytic approaches and some additional considerations. Stat Med. 1993;12(14):1293-1316.

14. Jaeschke R, Guyatt GH, Sackett DL. Users' guides to the medical literature. III. How to use an article about a diagnostic test. B. What are the results and will they help me in caring for my patients? The Evidence-Based Medicine Working Group. JAMA. 1994;271(9):703-707.

15. Estrada JJ, Petrosyan M, Barnhart J, Tao M, Sohn H, Towfigh S, Mason RJ. Hyperbilirubinemia in appendicitis: a new predictor of perforation. J Gastrointest Surg. 2007;11(6):714-718.

16. Khan S. Elevated serum bilirubin in acute appendicitis :a new diagnostic tool. Kathmandu Univ Med J (KUMJ). 2008;6(2):161-165.

17. Kaser SA, Fankhauser G, Willi N, Maurer CA. C-reactive protein is superior to bilirubin for anticipation of perforation in acute appendicitis. Scand J Gastroenterol. 2010;45(7-8):885-892.

18. Atahan K, Ureyen O, Aslan E, et al. Preoperative diagnostic role of hyperbilirubinemia as a marker of appendix perforation. The Journal of International Medical Research. 2011;39:609-618.

19. Emmanuel A, Murchan P, Wilson I, Balfe P. The value of hyperbilirubinaemia in the diagnosis of acute appendicitis. Ann R Coll Surg Engl. 2011;93(3):213-217.

20. Hong YR, Chung CW, Kim JW, Kwon CI, Ahn DH, Kwon SW, Kim SK. Hyperbilirubinemia is a significant indicator for the severity of acute appendicitis. J Korean Soc Coloproctol. 2012;28(5):247-252.

21. McGowan DR, Sims HM, Zia K, Uheba M, Shaikh IA. The value of biochemical markers in predicting a perforation in acute appendicitis. ANZ J Surg. 2013;83(1-2):7983.

22. Chaudhary P, Kumar A, Saxena N, Biswal UC. Hyperbilirubinemia as a predictor of gangrenous/perforated appendicitis: a prospective study. Ann Gastroenterol. 2013;26(4):325-331.

23. D'Souza N, Karim D, Sunthareswaran R. Bilirubin; a diagnostic marker for appendicitis. Int J Surg. 2013;11(10):1114-1117.

24. Nomura S, Watanabe M, Komine O, Shioya T, Toyoda T, Bou H, Shibuya T, et al. Serum total bilirubin elevation is a predictor of the clinicopathological severity of acute appendicitis. Surg Today. 2014;44(6):1104-1108.

25. Socea B, Carap A, Rac-Albu M, Constantin V. The value of serum bilirubin level and of white blood cell count as severity markers for acute appendicitis. Chirurgia (Bucur). 2013;108(6):829-834.

26. Chambers AC, Bismohun SL, Davies H, White P, Patil AV. Predictive value of abnormally raised serum bilirubin in acute appendicitis: a cohort study. Int J Surg. 2015;13:207-210.

27. Muller S, Falch C, Axt S, Wilhelm P, Hein D, Konigsrainer A, Kirschniak A. Diagnostic accuracy of hyperbilirubinaemia in anticipating appendicitis and its severity. Emerg Med J. 2015;32(9):698-702.

28. Saxena D, Tandon M, Shah Y, Gedam BS. Hyperbilirubinemia as a diagnostic tool for the prediction of appendicular perforation: a prospective study. Euroasian J Hepatogastroenterol. 2015;5(2):87-89.

29. Shahabuddin SF, Khan S, Finan RI, Khan MA. Serum bilirubin and C-reactive protein as predictor of perforation in acute appendicitis. A prospective cohort study. Int J Scientific Research. 2016;5:158-161.

30. Abouzeid A, Saleh M, Zayed O, Awad M. Validity of serum total bilirubin as a diagnostic tool for acute appendicitis. International Journal of Surgery And Medicine. 2017;3:216-222.

31. Eren T, Tombalak E, Ozemir IA, Leblebici M, Ziyade S, Ekinci O, Alimoglu O. Hyperbilirubinemia as a predictive factor in acute appendicitis. Eur J Trauma Emerg 
Surg. 2016;42(4):471-476.

32. Cheekuri SR, Mohanty A, Ganesh T, Kannan R, Smile R. Hyperbilirubinemia as a predictor of the severity of acute appendicitis - an observational study. Int Surg J. 2017;4:1341-1344.

33. Vineed S, Hanumanth Naik RK. Diagnostic accuracy of hyperbilirubinemia in predicting perforated appendicitis. Int Surg J. 2017;4:3441-3444.
34. Bossuyt PM, Reitsma JB, Bruns DE, Gatsonis CA, Glasziou PP, Irwig L, Lijmer JG, et al. STARD 2015: an updated list of essential items for reporting diagnostic accuracy studies. BMJ. 2015;351:h5527.

35. Giordano S, Paakkonen M, Salminen P, Gronroos JM. Elevated serum bilirubin in assessing the likelihood of perforation in acute appendicitis: a diagnostic meta-analysis. Int J Surg. 2013;11(9):795-800. 